Z Gerontol Geriat 2015 · 48:494 DOI 10.1007/s00391-015-0910-3

Online publiziert: 8. Juli 2015

๑) Springer-Verlag Berlin Heidelberg 2015
Rosmarie Martin ${ }^{1} \cdot$ D. Schachtschabel ${ }^{2} \cdot$ W. Beier ${ }^{1} \cdot$ H. Martin ${ }^{1}$

${ }^{1}$ Leipzig, Deutschland

${ }^{2}$ Marburg, Deutschland

\title{
Stellungnahme zur Eliminierung des Max-Bürger-Preises der Deutschen Gesellschaft für Gerontologie und Geriatrie
}

Aus Alters- und gesundheitlichen Gründen konnten wir - die Unterzeichner dieses Schreibens - an dem Kongress und der Mitgliederversammlung in Halle nicht teilnehmen. So haben wir erst jetzt von der Abschaffung des Max-Bürger-Preises erfahren. Wir sind empört, dass erst 70 Jahre nach Kriegsende die Integrität des Gründers der Gesellschaft für Altersforschung, der gleichnamigen Zeitschrift und des Autors des Buches Altern und Krankheit - eines der klassischen Werke der Gerontologie - zur Diskussion gestellt wird, also zu einem Zeitpunkt, an dem die meisten Zeitzeugen verstorben sind. Dazu gehören die ehemaligen Mitglieder dieser Gesellschaft, die Professoren Ries, Leutert und Rotzsch der Universität Leipzig, die zugleich Schüler von Max Bürger gewesen sind. Mit ihnen verfolgten wir eine enge wissenschaftliche Zusammenarbeit und haben von ihnen gehört, dass Bürger ein vorbildlicher Arzt, Hochschullehrer und Mensch war. Auch haben ehemalige Mitarbeiter, Studenten und Patienten 1946 bezeugt, dass er gefährdeten Personen geholfen und Nichtarier behandelt hat. Deshalb wurde er nach seiner Entlassung aus dem Klinikdienst 1947 wieder als Professor und Klinikdirektor eingestellt. Das war eine sehr seltene Ausnahme in Ostdeutschland. Hier wurde viel schärfer mit ehemaligen Mitgliedern der Nationalsozialistischen Deutschen Arbeiterpartei (NSDAP) umgegangen. Im Gegensatz dazu wurde in Westdeutschland der von Ihnen angeführte Prof. Catel, berüchtigt für die Euthanasie an Kindern, nach seinem Weggang aus Leipzig 1947 in Wiesbaden als unbelastet eingestuft, 1949 in Hamburg beim Entnazifizierungstribunal freigesprochen und 1954 als Professor für Kinderheilkunde in Kiel wieder eingestellt.

Das von Ihnen angeführte Gutachten für die Habilitationsarbeit des Dr. Ernst Otto Berger aus dem Jahr 1945 haben wir eingesehen. Der Titel dieser Arbeit lautet: „Methodik und kritische Auswertung der Encephalographie zur Erfassung pathologisch-anatomisch nachweisbarer Hirnanomalien im Kindesalter". Es gibt keine Hinweise auf menschenverachtende Versuche in dieser Arbeit, sodass Bürger keinen Grund gehabt haben dürfte, die Erstellung des Gutachtens abzulehnen. Sein Gutachten ist rein wissenschaftlich verfasst, frei von jeglichen politischen Implikationen. Allen Kollegen, die sich nicht „in zwei totalitären Staaten durchlavieren mussten“ (Zitat: S. 625), möchten wir sagen, dass schon viel Zivilcourage dazu gehörte, sich dem politischen Druck zu widersetzen. Bürger trat übrigens erst 1937 in die NSDAP ein, was dafür spricht, dass er kein Hurra-Patriot war. Wahrscheinlich hätte er bei Verweigerung der Mitgliedschaft die Klinikleitung sowie die Forschung aufgeben müssen und sich damit nicht mehr für Mitarbeiter und $\mathrm{Pa}$ tienten einsetzen können. Auch in der Deutschen Demokratischen Republik (DDR) war eine wissenschaftliche Karriere ohne Mitgliedschaft in der Sozialistischen Einheitspartei Deutschlands (SED) fast unmöglich. Wir sind der festen Überzeugung, dass Max Bürger nicht nur als Arzt und Wissenschaftler, sondern auch als Mensch sich vorbildlich in den zwei totalitären Systemen verhalten hat. Es ist uns rätselhaft, aufgrund welcher objek- tiven Kriterien die Mitglieder der Deutschen Gesellschaft für Gerontologie und Geriatrie (DGGG) zu ihrem negativen Urteil bezüglich des Max-Bürger-Preises gelangt sind.

Wir erwarten deshalb eine plausible Begründung der DGGG bzw. des Präsidiums dazu.

Dr. rer. nat. R. Martin, ehemalige Stellvertretende Vorsitzende der Sektion I der DGGG

Prof. Dr. med. habil. D. Schachtschabel, Ehrenmitglied, ehemaliger Vizepräsident und Vorsitzender der Sektion I der DGGG

Prof. Dr. phil. habil. W. Beier, Direktor des Instituts für Biophysik der Universität Leipzig von 1960-1982, Träger des MaxBürger- und des Virchow-Preises, Mitglied der Leopoldina, wissenschaftliche und persönliche Kontakte zu M. Bürger

PD Dr med. habil. H. Martin, ehemaliger Vorsitzender der Sektion I der DGGG

\section{Korrespondenzadresse}

Dr. rer. nat. R. Martin

Händelstr. 15, 04288 Leipzig

dr.hans.martin@gmx.de

Interessenkonflikt. R. Martin, D. Schachtschabel, W. Beier, H. Martin geben an, dass kein Interessenkonflikt besteht.

Siehe hierzu den Bericht der Präsidentin, Prof. A. Hedtke-Becker und des Past-Präsidenten, Prof. A. Simm, Z Gerontol Geriatr 7/2014. S. 623-625. 